\title{
Investigando o processo psicolingüístico de tradução: identificação de problemas de tradução e possíveis soluções por meio de estratégias
}

\section{Regina Maria Guarnier Domiciano \\ UEL}

Este trabalho tem por objetivo apresentar uma análise parcial do processo psicolinguiístico da tradução de cartas em inglês escritas por George Craig Smith, funcionário da Companhia de Terras Norte Paraná, companhia responsável pela colonização do norte velho do Paraná. O processo psicolinguiístico de tradução pode ser entendido como um conjunto de problemas para os quais o tradutor procura encontrar soluções, através de estratégias. Na primeira fase, para o resgate do processo lingüístico da tradução das cartas foi utilizada a técnica do pensamento em voz alta. Três sujeitos, alunos do Curso de Letras Anglo-Portuguesas, participaram dessa fase da pesquisa. $\mathrm{Na}$ segunda fase, foram identificados e analisados os problemas de tradução assim como as estratégias para solucionar os mesmos.

The aim of the research was to present a partial analysis of the psycholinguistic process of the translation of letters written by George Craig Smith, an employee of the Companhia de Terras Norte Paraná which was responsible for the colonization of that area. The psycholinguistic process under investigation illustrates the problems of translation for which the learner tries to find solutions for using strategies. The first phase of this research consisted of obtaining the process of translation by applying the think-aloud protocol. Three students, undergraduates majoring in English and Portuguese, were the subjects of this research. During the second phase, the problems of translation, in addition to the strategies applied, were identified and analyzed. 


\section{Introdução}

George Craig Smith foi um dos primeiros funcionários da Companhia de Terras Norte do Paraná (subsidiária da Paraná Plantation Limited), empresa responsável pela colonização desta região, e trabalhou na Companhia de 1925 a 1937, com interrupções. Em 1975 volta a residir em Londrina, e, em dezembro de 1984, é homenageado pela Câmara Municipal de Londrina, sendo, então, considerado um dos pioneiros da colonização. George Craig Smith correspondeu-se com seus familiares e também com seus ex - colegas de trabalho da Companhia. Embora fosse brasileiro, a maioria das cartas foi escrita em inglês, pois era filho de pais descendentes de ingleses (ingleses radicados em São Paulo). As cartas registraram acontecimentos do cotidiano, reflexões e relatos sobre a colonização do norte novo do Paraná, lembranças outras que a memória insistiu em guardar, vindo a resgatar a trajetória de uma vida. O conteúdo das cartas é também permeado de uma visão particular sobre os acontecimentos da época, os momentos de lazer, as comemorações em família e as sociais, além do registro das emoções sobre os imprevistos bem-vindos ou frustrantes, as boas e as más notícias. Algumas dessas cartas, já arquivadas como documentos, vão constituir o corpus para a pesquisa do processo de tradução.

A tradução das marcas de um estilo epistolar (sua linguagem informal, a manifestação de diversos graus de intimidade) se formaliza em procedimentos técnicos de tradução. Aubert (1998: 105-110) apresenta os seguintes tipos de modalidades de tradução: omissão, transcrição, empréstimo, decalque, tradução literal, transposição, explicitação, modulação, adaptação, erro, correção e acréscimo. É possível recuperar essas modalidades de tradução analisando o produto final, o texto traduzido, mas o processo de tradução é um acontecimento que se dilui no fugaz processo psicolinguístico. Tal processo é constituído de deslocamentos cognitivos, e é a complexidade desses deslocamentos que forma uma base para um possível produto, mesmo que não seja definitivo. Para Lörscher (1991), a investigação desse processo é importante, pois só com base nos estudos empíricos da tradução, usando um processo analítico, é que podemos elaborar hipóteses sobre o que está passando na mente do tradutor, seja ele aprendiz ou profissional, e também porque os estudos empíricos irão nos proporcionar uma compreensão sobre o ato de traduzir, sobre aspectos dos processos 
mentais de recepção do texto em língua de partida (LP) e da produção do texto em língua de chegada (LC) e sobre as estratégias mentais utilizadas pelo tradutor. Para o referido autor (idem: 94 - 96), o problema de tradução é um problema linguístico que é identificado ou na fase da recepção, ou na fase da produção ou na fase recepção/produção. As estratégias de tradução são procedimentos que os tradutores usam para resolver os problemas de tradução, ativando-as ao reconhecer um problema e suspendendo-as quando encontra a solução preliminar ou quando não as considera adequadas para solucionar os problemas de tradução.

\section{Processo psicolingüístico da tradução}

Embora a tradução possa ser investigada como produto, o objetivo deste trabalho é investigar o processo psicolingüístico de tradução, visto que este constitui uma etapa essencial para se chegar ao produto. Dado a natureza do processo, há consenso de que o sujeito que traduz (no nosso caso do português para o inglês), o tradutor aprendiz ou profissional, não produz um texto "impecável" em LC já na primeira etapa, pois encontra problemas e usa estratégias de diferentes tipos para resolvê-los. As etapas para se chegar a soluções e, até mesmo, reorganizar as soluções quando consideradas não satisfatórias são os passos que vão constituir o processo psicolinguiístico da tradução. O presente trabalho pretende enfocar a configuração das fases, das estratégias e das soluções encontradas para problemas de tradução por alunos do curso de Letras que participam de projeto de tradução.

Para esse tipo de investigação, a primeira fase seria identificar os problemas de tradução e as possíveis estratégias que têm lugar ao se traduzir, isto é, quando o sujeito, verbalizando possíveis equivalências semânticas, textuais, de gênero, etc, minimiza o uso de filtros de regras gramaticais e textuais (regras de coesão e coerência) e faz fluir um "texto" com interrupções, reformulações, com considerações sobre decisões tomadas, questionamento dessas decisões, entremeado de silêncios. Esse "texto" é considerado um texto que espelharia o processo denominado processo psicolingüístico. A primeira parte deste trabalho constituirá da exposição da primeira fase do ato tradutório: os problemas de tradução, as soluções dos problemas de tradução ou até mesmo as fases 
intermediárias para se chegar à solução do problema de tradução e as estratégias que o sujeito emprega nessa fase exploratória. A segunda etapa se constituirá da análise e classificação feitas segundo as categorias apresentadas por Lörscher (1991). Com base nesse levantamento, será apresentado um perfil exploratório do aprendiz de tradução quando ele não frequienta um curso de tradução, mas recebe formação numa área relacionada mas que pode também estar num processo de traduzir com autonomia (Alves, Magalhães \& Pagano, 2000).

\section{Metodologia}

A investigação dos processos psicolingüísticos realizados por um sujeito é um campo recente de pesquisa. Erickson \& Simon (1980) argumentam a validade de relatos verbais ser considerados "dados" para a pesquisa na Psicolingüística; Faerch e Kasper (1987) explicam a natureza dos métodos de introspecção além de apresentarem argumentos a seu favor; Tirkkonen-Candit (1998), Séguinot (1996), Fraser (1996), Lörscher (1991) e Pagano (2001) fizeram pesquisas desses processos usando a técnica do protocolo - gravação do pensamento em voz alta. Essa técnica, assim como a elaboração de diário, têm a possibilidade de investigar os problemas de tradução e as estratégias para solucionar os problemas que o sujeito tradutor apresenta. Esse sujeito pode ser o tradutor profissional ou o que está em aprendizagem.

Segundo Lörscher (1991: 54 -55), estas são algumas características do processo do pensamento em voz alta quando utilizado para resgatar o processo psicolingüístico da tradução:

- os sujeitos só reportam o que está no foco de atenção deles;

- think-aloud é um tipo de verbalização comum;

. o processo de tradução opera em dados verbalmente codificados ou pausas;

. os problemas do tradutor e o que ele pensa em voz alta quando se confronta com tais problemas são analisados;

- a solução dos problemas de tradução é sempre realizada através de uma série de passos; geralmente, os sujeitos não alcançam imediatamente as soluções que consideram ser adequadas. 
A técnica do protocolo-gravação em voz alta usada consiste em primeiramente o sujeito fazer um warm-up, depois traduzir o texto em voz alta e gravando a tradução verbalizando os processos mentais que utiliza para resolver os problemas de tradução. Depois, é feita a transcrição das gravações, seguindo uma anotação apropriada:

$$
\begin{aligned}
& \text {... Para uma pausa breve } \\
& \text { (não-inteligível) } \\
& \text { (repetições) } \\
& \text {...... Para pausa longa } \\
& \text { I Para pausa de mais de um minuto } \\
& \text { (hesitações) }
\end{aligned}
$$

Segundo Lörscher (1991), estratégias de tradução são procedimentos que os sujeitos empregam para resolver problemas de tradução. As estratégias são acionadas quando o sujeito diagnostica um problema e a solução que o sujeito / tradutor encontra ou se não encontra nenhuma solução. Lörscher propõe 22 categorias de problemas de tradução no livro Translation Performance, Translation Process and Translation Strategies. Segundo esse mesmo autor, "problema de tradução" é um conceito que engloba todos aqueles problemas lingüísticos que o sujeito tem que resolver quando realiza uma tradução (idem: 94). Para o nosso trabalho, seguiremos a classificação de Lörscher (idem: 95) segundo a qual os problemas de tradução podem ser:

a) Problema de recepção que ocorre quando o sujeito tem dificuldade em compreender o segmento textual da língua de partida (daqui em diante LP);

b) Problemas de produção que ocorrem quando o sujeito tem dificuldades em encontrar segmentos de textos / palavras que considera equivalentes na LC. Os procedimentos adotados para se chegar ao texto equivalente são dois:

- separar o sentido das formas do segmento de texto da LP e o sentido que vai ser a base para procurar a equivalência.

- procurar o segmento de texto equivalente com base nas formas dos segmentos de texto da LP.

c) Problemas de recepção / produção que ocorrem quando o sujeito tem dificuldades tanto na recepção quanto na produção de um 
segmento da LP em texto equivalente em LC.

Três alunas do curso de Letras Anglo-Portuguesas (uma do $3^{\circ}$ ano e duas do $4^{\circ}$ ano) participaram da pesquisa como sujeitos. Na análise foram denominadas $\mathrm{A}, \mathrm{B}$ e $\mathrm{C}$ respectivamente. As alunas escolheram aleatoriamente um parágrafo de uma das cartas, preparavam o gravador e usando a técnica do protocolo - gravação do pensamento em voz alta, gravavam a tradução. Depois, o texto traduzido gravado foi transcrito conforme a anotação apropriada. Feito isso, os dados foram entregues para a coordenadora do projeto que os analisou e os classificou conforme as categorias propostas por Lörscher. No corpo do trabalho, apresentaremos exemplos, e, no Anexo 1, o levantamento completo dos dados. As seguintes siglas foram usadas para classificar as etapas dos processos psicolingüísticos: pt (problema de tradução); spt (solução de problema de tradução); $v p t$ (verbalização do problema de tradução); sppt (solução preliminar para o problema de tradução); sppt (solução preliminar para problema de tradução a,b...); mst (monitoramento do segmento de texto); $p t->$ ref $1,2 \ldots$ (refraseando segmentos de texto a, b...).

\section{Identificação de problemas de tradução e análise das estratégias}

Problemas de tradução e estratégias para a solução

1) Percebendo um problema de tradução (pt). O problema está relacionado com a tradução de um segmento de texto (palavra, parte da sentença) da LP para LC.

Documento n. ${ }^{\circ} 2149$

A) She is the pitcher - Jonathan.

Ela é a

......

$\mathrm{pt} / \mathrm{ps}$

a arremessadora.

$\mathrm{Spt}$

2)Verbalizando um problema de tradução (vpt). O sujeito verbaliza o problema relacionado com uma palavra ou um segmento do texto na LC. Eles podem ser verbalizados antes ou depois da solução. 
Documento n. ${ }^{\circ} 2308$

B

... I did not sleep well but I have put my sleep up to date!

Mas ........ pt

a idéia seria dormir bastante para compreender todo o tempo

em que ele não podia dormir ...

devido ao desconforto causado pelo clima ...

e pela não ...... confortável acomodação vpt

hum...colocarei meu sono em dia.. $\quad \mathrm{spt}$

3) Procurar por uma solução (preliminar) para um problema de tradução. Começa logo depois da percepção do problema.

Documento n. ${ }^{\circ} 2274$

A

... and the wily Schaff who

1 pt

uma dúvida de vocabulário / vpt

procurei no dicionário à pspt

hã tá ..... ( sem verbalização)

e pelo esperto Schaff spt

Documento n. $^{\circ} 2149$

C

... just finished their soy bean crop

e acabaram de terminar a colheita de feijão

(soy bean)

soja... feijão

deixa eu procurar no dicionário

de feijão, soja é ...

colheita de soja

$\mathrm{pt}$

mspt

vspt

vspt

$\mathrm{spt}$

4) Às vezes há uma série de soluções preliminares sem uma solução definitiva que virá "reescrevendo o texto".

Documento n. ${ }^{\circ} 2290$

B

We had the pleasure of shaking hands with ... 
Nós tivemos o prazer de

…..

de apertar a mão

pt ( sem verbalização)

/

sppt a

de dar as mãos

à $p s$

(suspensão)

sppt b .....

5) Monitoração do segmento de texto em LP: O sujeito repete verbatim ou segmentos do texto que verbalizou, mas fazendo uma adequação em relação à LC porque o sentido não é adequado em relação à LP Documento 1. ${ }^{\circ} 2275$

A

And official invitation in due time. um convite oficial no tempo certo .

.......... mst (monitoração do segmento de texto em LP sem verbalizar)

no devido tempo

spst na LC.

6) Refraseando segmentos de texto em L2: o sujeito retorna a segmentos de texto em L2 e verbaliza de forma diferente o texto em $L 2$ pois parece que não aceita o segmento como um equivalente adequado.

Documento n. ${ }^{\circ} 2149$

A

it made me want to see you again ...

fez me sentir

...

fez me sentir com vontade de

$\mathrm{pt}$

....

de querer vê- lo

spt com repetição

ptà ref a

(........

pt

querer vê-los novamente de novo

spt com ref a

......

mspt

năo

I

cancelamento de ref a

.........

ver vocês todos novamente

sà ref

spt com ref b 
Com base no corpus escolhido para estudar os processos psicolinguísticos do ato de tradução, os dados apontam que os tipos de problemas de tradução que os aprendizes de tradução apresentam com mais frequiência são os problemas de tradução de palavras, isto é, são problemas pontuais de tradução tanto na recepção quantos na produção. A estratégia mais verbalizada e mais frequiente para solucionar o problema de tradução é a procura da palavra no dicionário. Essa estratégia parece ser o que os sujeitos consideram como eficiente para solucionar o problema de tradução, pois os três sujeitos usaram-na e não suspenderam a solução do problema de tradução dada pelo dicionário.

Quando a solução para o problema de tradução se torna textualmente incoerente seja por incoerência ou inadequação sintática, várias são as soluções para equivalência textual ou aceitabilidade do texto em LP. Nesses casos, a solução do problema de tradução pode passar por monitoramento ou refraseamento de um de segmento de texto. A estratégia mais empregada é a da monitoração do segmento em LC. O aprendiz refaz, desmancha o seu trabalho psicolingüístico para encontrar uma adequação em LC, mas nem sempre é bem sucedido. Ele tem a tendência de suspender o processo, mas certamente terá de retomá-lo e encontrar uma solução, nem sempre a melhor, para apresentar o texto final.

As soluções para os problemas de tradução parecem fornecer uma pista para trabalhar uma co-relação entre solução de problemas de tradução e os procedimentos técnicos de tradução, cujo objeto de análise é o produto. Quando os sujeitos da pesquisa, durante o processo de identificação do problema de tradução sem verbalização, propõem a solução de problema de tradução numa sequiência, isto é, sem interrupções ou repetições, há uma incidência do procedimento técnico de tradução literal. Na medida em que há monitoramento e refraseamento há uma tendência para tradução oblíqua, com a possibilidade da presença mais significativa dos seguintes procedimentos de tradução: transposição, modulação e acréscimo.

\section{Conclusão}

A análise qualitativa dos dados aponta que as categorias de análise que Lörscher (1991) propõe para os problemas de tradução são adequadas para a análise de estratégias e de problemas linguiísticos de tradução mesmo quando o sujeito da tradução ainda está em fase de aprendizagem. 
Na primeira fase do processo psicolingüísticos das traduções de cartas, identificamos problemas de tradução e suas soluções verbalizadas pela técnica do protocolo em voz alta. Esses problemas se enquadram nas 3 categorias propostas pelo autor: problemas de recepção, problemas de produção e problemas de recepção / produção. A análise também corroborou a definição de Lörscher para os problemas lingüísticos de tradução em sujeito que não tem formação específica para ser tradutor.

Esses problemas são principalmente de natureza pontual, isto é, de vocabulário, palavras cujos sentidos o aprendiz tem dificuldade em diagnosticar, palavras que embora ele saiba um dos seus sentidos, este não é adequado ao contexto, e, palavras que na LP, ele sabe o sentido mas tem dificuldade em encontrar o termo adequado correspondente na LC, isto é, o sistema paradigmático de equivalência não está automaticamente ajustado para encontrar o termo equivalente adequado ao gênero epistolar que se caracteriza pelo emprego de certas palavras e estruturas frasais que pertencem ao padrão coloquial. Outro tipo de problema é de adequação da estrutura frasal. O sujeito parece saber a correspondência de sentidos, mas parece que, devido a uma sobrecarga na elaboração semântica, não consegue elaborar a estrutura adequada aceitável em LC. Quando o leque de possibilidades de solução para o problema de tradução se amplia, a tendência é suspender e voltar ao mesmo na revisão do texto, quando obrigatoriamente uma solução, mesmo não sendo a melhor, terá de ser apresentada.

Com base nesses dados, o perfil do tradutor aprendiz que não está recebendo uma formação específica sobre Tradução aponta que há necessidade de automatizar muitas equivalências entre LP e LC, além da necessidade de explorar a internalização de outras estratégias para ampliar o número de opções que ele pode dispor quando em ato de traduzir. Esses resultados de análise nos estimulam a usar o mesmo modelo para criar uma tipologia de estratégias de tradução e uma tipologia de textos para tradução. Visto que nessa pesquisa participaram apenas três sujeitos; outras pesquisas, usando a mesma metodologia, poderiam ser realizadas para corroborar os dados e as conclusões obtidas.

\section{Referências bibliográficas}

ALVES, Fábio. Lançando anzóis: uma análise cognitiva dos processos mentais em tradução. Revista de Estudos da Linguagem, v. 4, n. 2, p. 
71-90, 1996.

AUBERT, Francis Henrik. Modalidades de tradução: teoria e resultados. Tradterm. v. 5, n. 1, p. 99-128, 1998.

ERICKSON, K.A. \& SIMON, H.A.Verbal reports as data. Psychological Review,.n. 87, p. 215-251, 1980.

FRASER, Janet. The Translator Investigated: Learning from translation analysis. The Translator. v. 2, n. 1, p. 65-79, 1996.

LÖRSCHER, W. Translation Performance, Translation Process, and Translation Strategies. Tübine, Narr, 1991.

PAGANO, Adriana, MAGALHÃES, C. \& ALVES, Fábio. Traduzir com autonomia: estratégias para o tradutor em formação. São Paulo: Contexto, 2000.

PAGANO, Adriana (Org.). Metodologia de Pesquisa em Tradução. Belo Horizonte: Fac. de Letras, UFMG, 2001.

SÉGUINOT, C. Some thoughts about think-aloud Protocols. Target. v. 8, n.1, p. 75- 95, 1996.

TIRKKONEN-CANDIT, S. Who verbalizes what: a linguistic analysis of TAP texts. Target, v. 9, n. 1, p. 69-84, 1998.

\section{ANEXO 1}

Tipo pt - spt (sem verbalização)

Doc 2316

A

and they also have their own restaurant

e eles têm

.......

pt

restaurante próprio

pt

Doc 2337

B

full of hate and lies

cheia de

......

ódio e mentiras

pt

Doc 2701

C

spt 
be as it may

$\mathrm{pt}$

seja como for spt

Tipo pt com verbalização para encontrar a solução : pt ( verbalização de problema de recepção)

Doc 2323

spt

A

in spite of so many drawbacks

apesar de tantos

.......

deixa eu ver no dicionário

$\mathrm{pt}$

para spt

/I

hã..ta

desencontros

spt

Doc 2367

A

he has to use magnifying glass

já que ele tem que usar

...... magnifying glass

segmento de texto em LP

pt com repetição do

vou procurar no dicionário

verbalização da estratégia

para spt

/I

magnifying glass como lupa ... então ficaria verbalização da solução do pt (produção)

já que ele tem que usar uma lupa spt

Doc 2363

$\mathrm{C}$

Faded

.......

a maioria das gravuras.. fotos ... faded

$\mathrm{pt}$

de recepção)

refr/ repetição ( pt 
vou procurar no dicionário o que seja esse termo estratégia de solução para pt ( recepção)

//

não encontrei nenhuma tradução conveniente pra verbalização para spt de recepção

faded nesse contexto ... mas... acredito que seja

alguma coisa como deteriorada

\section{......}

apagadas por se tratar de fotos de tempos atrás

sppt a

Doc 275

B

I had a touch of cold

eu tive

...... touch...

pt com repetição do

segmento de texto em LP

toque...

sppta

nesse contexto talvez ficasse melhor

um ameaço de gripe spt com verbalização para encontrar solução em de LC (produção)

Doc 274

B

the wet season

...wet

... molhado

pt com repetição

... nesse contexto...

sppt a

outra spt

estação das chuvas verbalização da procura de

Doc 2308

B

I am enclosing

eu 
......

I (pesquisa no dicionário para certificar

do sentido de enclose)

deveria ser traduzido como anexar

sppta

eu estou anexando

pt

\section{sppta}

ref spta

Doc 2364

C

Infliching

eu vou pesquisar no dicionário

$\mathrm{pt}$

verbalização da

estratégia para solução de pt de recepção

...hum... não encontrei em nenhum

dos dicionários que eu pesquisei

spt suspensa

talvez fosse alguma coisa como grande

coragem

não sei

talvez como uma coragem destemida

uma bravura destemida...

talvez fique meio redundante

mspt a

mspt b

(suspensão da solução)

Problema de tradução com verbalização de monitoração em LP na solução do problema

Doc 2308

$\mathrm{B}$

I have just received a letter

eu também recebi uma carta

bem...deixe pensar melhor...

tem um present perfect aqui

pt

...... melhor

eu acabei de receber uma carta

pt com verbalização

mspt emLP 


\section{Doc 2361}

B

to the Brazilian part of the colonization

a parte brasileira

eu acredito quer ficaria melhor

pt

se traduzisse

monitoração do segmento

em LP para spt

a parte da colonização feita por brasileiros

spt a

a parte que os brasileiros têm na colonização

spt b

Doc 2361

B

and have published in all newspapers

e publicar em todos os jornais

acredito que ficaria melhor

verbalização da monitorição do segmento de texto em LP para spt fazer publicar em todos os jornais spt

Doc 2361

B

special conversation

ele põe "a special conversation"

verbalização do $\mathrm{pt}$

mas eu acho que simplesmente

monitoração do

segmento de texto em LP para spt

eu tive uma conversa

spt

Doc 2701

A

All is stale

Tudo está

aqui ela fala all is stale

$\mathrm{pt}$

... acredito que queira dizer...

verbalização do $\mathrm{pt}$ spt 
os acontecimentos não tem nada de novo ......

eu traduziria como tudo está sem graça segmento de texto de ...spt a ou... nada tem graça

Doc 2701

A

as regards the splendid colonization of the britchers

com relação a colonização explêndida pelos britânicos

......

sobre a explêndida colonização pt sem verbalização mspt em LP

Doc 2364

$\mathrm{C}$

as to the photos you asked me to send you

Como as fotos que você me pediu

spt

......

a respeito das fotos que você me pediu

mspt em LP

\section{Problema de Tradução com monitoração em $\mathbb{L C}$ na solução do problema}

Doc 2704

B

with the terrific price of the dollar com o

...

terrivel preço do dólar

pt

talvez ficasse melhor

com o exorbitante preço do dólar

spta

mspt

sptb

Doc 2257

$\mathrm{C}$

I am afraid the pictures have gone astray

Estou organizando as idéias

de tradução

verbalização de estratégia 
rece... receio que as figures tenham

tenho uma dúvida de vocabulário aqui astray verbalização do pt

provavelmente desviado no correio

sppta

a alguma coisa assim ...

mspt em LP/LC

procurei no dicionário astray...

tá... desviar então

mspt b

receio que as fotos foram desviadas no correio

manutenção de

sptb com ref de segmento de texto em LP

...é... durante a postagem

Doc 3700

B

shake hands

de apertar a mão

$\mathrm{pt}$

a não...

com monitorição em LC

de dar as mãos com

spt b

não estou entendendo aqui...

suspensão de spt em LC

Refrasendo o segmento de texto dasolução para o problema de tradução em LC

Doc 2248

A

You will feel mentally different on your return

Você se sentirá

......

mentalmente diferente quando voltar

pt

em melhores condições de saúde

spta

suspensão de spt

Doc 2337

B

in publishing without permission your picture

em publicá-lo sem permissão

.......

mst da spt em LC

em publicar sem permissão a fotografia

ref spt 
Doc 2338

A

which you remember clearly

...

observando recordações claras...

pt

se recorda tão claramente

se recorda muito bem

$\begin{array}{ll}\text { spt } & \\ \text { ref sta em LC } \\ \text { ref stb em LC }\end{array}$

Doc 2345

A

attached is a copy of his speech he

authorized me to use it

I $\mathrm{pt}$

cuja cópia segue em anexo conforme pedido dele alías..

verbalização de mspt

cuja cópia segue em anexo conforme

autorização dele

ref st em LC

Doc 2313

$\mathrm{C}$

Therefore plenty of work

Então

......

há um monte de trabalh

melhor

há muito trabalho

pt

spt

verbalização de mspt

ref spt em LC

Doc 2321

$\mathrm{C}$

not being satisfied with my narrative

não estando satisfeito com minha narrativa

insatisfeito com minha narrativa

mspt

ref spt em LC

Doc 2327 
C

but what I most resent

mas os que me dá

...

mais raiva

$\mathrm{pt}$

o que mais me magoa

spt

ref stpt em LC

Doc 2327

C

we had a good talk

nós tivemos uma boa conversa

ou

mspt

nós conversamos bastante

ref spt em LC

Doc 2242

C

my special thanks to

meus especiais agradecimentos

estou especialmente grato

mspt

ref spt em LC 